\title{
Magnetic Properties of Nickel Phosphorus Films Prepared by Electroless Plating with Electrolysis
}

\author{
Hiroshi MATSUBARA*, Kazuhito MATSUKAWA, \\ Kazuhiro TSURU and Akifumi YAMADA
}

Received November 24, 1992 ; Accepted January 14, 1993

\begin{abstract}
Changes in the magnetic properties of nickel phosphorous films fabricated by electroless plating with simultaneous electrolysis were studied. The saturation magnetization decreased with potentiostatic cathodic polarization. This was due to an increase in the phosphorous content of the films upon the application of electrolysis. No distinct change was observed in the coercivity and crystallinity. The nickel phosphorous films formed by this process seemed to have less segregated structure compared with those formed by electroless plating. Saturation magnetization also changed upon the application of a pulsating current. The simultaneous application of electrolysis is, therefore, very effective in controlling the magnetic properties of nickel phosphorous films.
\end{abstract}

\section{INTRODUCTION}

Magnetic films are widely used for recording devices and sensors. Both dry processes, such as sputtering and evaporation, and wet processes, such as platings, are used as methods for fabricating the films. The wet processes have the advantage of high producibility. Especially, electroless plating has the capability to metalize non-conducting materials as do the dry processes. Moreover, it has a high throwing power. Electroless plating, however, also has the disadvantages of limitation of deposition elements compared with electroplating and a difficulty in controlling the properties of the film because of the many empirical factors involved in the relationships be-

Department of Chemistry, Nagaoka University of Technology, 1603-1 Kamitomiokamachi, Nagaoka, 940-21

Key words: electroless plating, electrolysis, nickel phosphorous alloy, magnetic properties tween the plating conditions and the properties of the films.

This paper is concerned with the magnetic properties of NiP films fabricated by electroless plating using simultaneous electrolysis. 1)2) The purpose of this study is to clarify the possibility of overcoming the latter disadvantage by showing the change in the magnetic properties of the films. The merit of electroless plating -its capability to metalize non-conducting materials - still exists in the new process. The present authors report the results of fundamental research on the NiP film, the most basic magnetic film formed by electroless plating 3)-6), prepared by electroless plating using simultaneous electrolysis in D.C. or a pulsating current.

\section{EXPERIMENTAL}

\subsection{Preparation of NiP films}

The composition and operating conditions of the standard plating bath were similar to those in References 2 and 6. The concentra- 


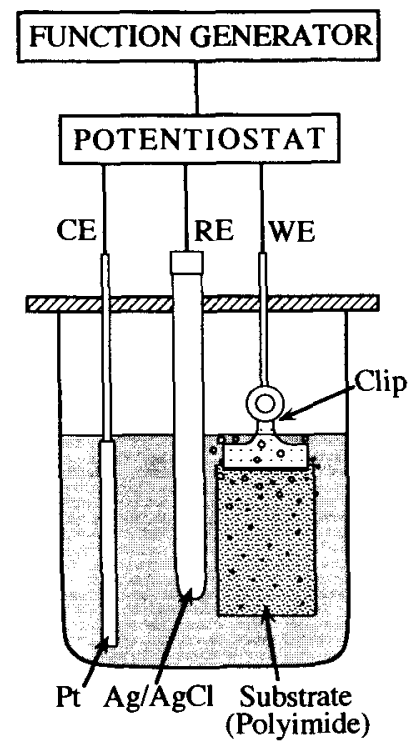

Fig. 1 Experimental apparatus for electrolessplating with electrolysis.

tions of nickel sulfate and sodium hypophosphite were varied in some cases. Polyimide film (Toray-Du Pont, Kapton100H) was used as the substrate. Thickness of the NiP films was $0.5 \mu \mathrm{m}$. The electroless plating with electrolysis was performed using the apparatus shown in Fig. 1. The substrate was fixed by a paper clip coated with epoxyresin except for the clip edge, as is illustrated in the scheme. A potentiostat (Hokuto Denko, HA-301) and a Punction generator (Hokuto Denko, HB-104) were used for electrolysis. After the substrate had been activated with an $\mathrm{SnCl}_{2}-\mathrm{PdCl}_{2}$ solution, electroless-NiP-plating was performed until the deposition potential became constant. Then a controlled potential was applied immediately. The electrolysis was performed potentiostatically with D.C. and a pulsating current(P.C.). In the electrolysis with the pulsating current, a controlled-potential pulse of $-100 \mathrm{mV}, 0.05$ second for each 5 seconds, was applied. The voltage applied was based on the rest potential (mixed potential) of the electroless plating.

\subsection{Measurement and analysis}

The magnetic properties were measured with a vibrating sample magnetometer (Riken Denshi, BHV-20). The crystallinity of the films was determined with an $\mathrm{X}$-ray diffractometer (Rigaku Denki, RAD IIIA). The compositions of the films were analyzed by determining the phosphorous content by the use of an inductively coupled plasma-emission spectrometer (Seiko Denshi, SPS-1200AR) and by means of the molybdenum-blue method.

\section{RESULTS AND DISCUSSION}

\subsection{Application of D.C. electrolygia}

\subsubsection{Deposition rate}

The relationships between the deposition rate and the applied voltage are shown in Fig. 2. The potential versus the mixed potential is plotted as the abscissa; the negative value indicates cathodic electrolysis. The electroless plating is shown as the potential of $0 \mathrm{mV}$. The deposition rate increased with the increase in the cathodic voltage for all nickel-sulfate concentrations. In the case of $0.14 \mathrm{~mol} \mathrm{dm}^{-3}$ of nickel sulfate, for example, the value increased from $19 \mu \mathrm{m} \mathrm{hr}{ }^{-1}$ for electroless plating to $35 \mu \mathrm{m} \mathrm{hr}^{-1}$ for an applied voltage of $-700 \mathrm{mV}$. Although the current increased slightly through the electrolysis, the apparent current efficiency was ca.45\%, as estimated from the increase in the deposition rate upon electrolysis. The mixed

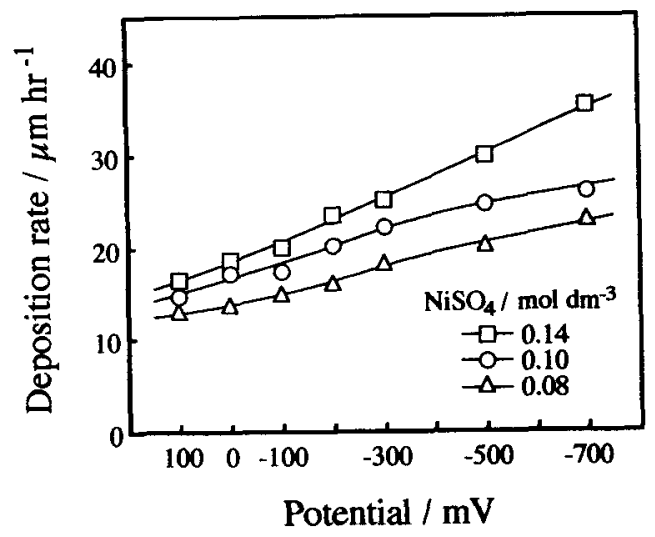

Fig. 2 Dependence of deposition potential on deposition rate of NiP films plated from various baths. 
potential of the electroless plating was ca. $-890 \mathrm{mV}$, almost independent of the $\mathrm{NiSO}_{4}$ concentration. It was difficult to examine any anodic polarization larger than that shown in the figure because the deposition became very slow.

\subsubsection{Magnetic properties of the films}

The change in saturation magnetization upon electrolysis is shown in Fig. 3. The value became much lower with the application of the cathodic electrolysis in all $\mathrm{NiSO}_{4}$ concentrations. For example, the value, $190 \mathrm{emu}^{-1}$, for electroless plating de-

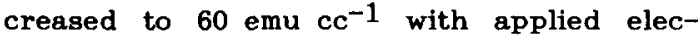
trolysis of $-700 \mathrm{mV}$ for the films plated from the bath with a $\mathrm{NiSO}_{4}$ concentration of $0.14 \mathrm{~mol} \mathrm{dm}^{-3}$. Moreover, the NiP films plated from the bath with a lower $\mathrm{NiSO}_{4}$ concentration, $0.08 \mathrm{~mol} \mathrm{dm}^{-3}$, became almost paramagnetic upon electrolysis with more than $-300 \mathrm{mV}$. The above results are plotted in Fig. 4 for various applied voltages as parameters. Although the saturation magnetization changed with the concentration of $\mathrm{NiSO}_{4}{ }^{6)}$, which was one of the bath factors, the slope of the curve decreased, indicating a decreasing dependence of the saturation magnetization on the bath factor. This can be simply interpreted by saying that a larger contribution of the electrolysis is

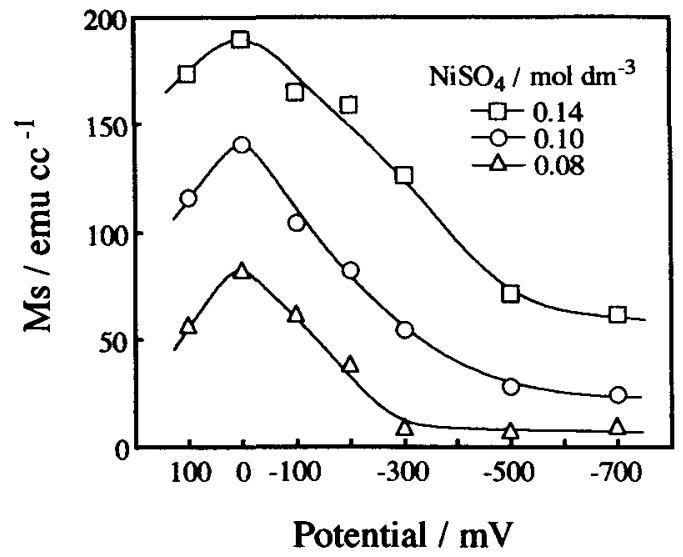

Fig. 3 Dependence of deposition potential on saturation magnetization of NiP films plated from various baths.

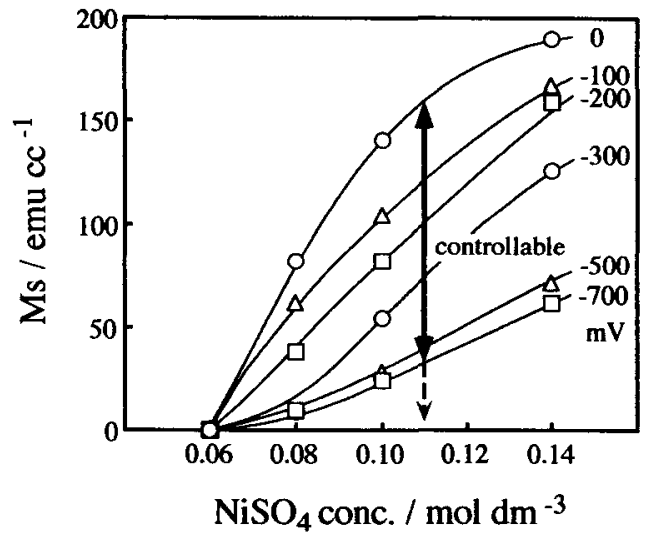

Fig. 4 Dependence of NiSO4 concentration on saturation magnetization of NiP films plated from various deposition potential.

observed in the plating with a larger polarization. The area indicated by the arrow shows the controllability of the saturation magnetization. The saturation magnetization of the NiP films was controllable, as has been described above, by applying electrolysis in the bath system introduced in this paper. The baths tend to get less stable when polarization progresses to more than ca. $1000 \mathrm{mV}$.

A similar investigation was performed on NiP films plated from the baths while the other bath factor, the concentration of sodium hypophosphite, was varied. In the electroless plating, the saturation magnetization can be changed with the concentration of sodium hypophosphite; however, the range of concentrations is rather limited because of the bath stability or the slow deposition rate. On the other hand, the saturation magnetization could be controlled over a wide range upon electrolysis, as is shown in Fig. 5. The range was from 38 to $160 \mathrm{emu} \mathrm{cc}-1$ and from 24 to $141 \mathrm{emu} \mathrm{cc}^{-1}$ for concentrations of 0.10 and 0.15 (standard bath) $\mathrm{mol} \mathrm{dm}^{-3}$ respectively. Figure 6 shows the coercivity for the NiP films plated from the standard bath. Coercivities of from ca.15 to 20 Oe appeared in all the NiP films; no distinct change was observed when electrolysis was applied. NiP films plated 


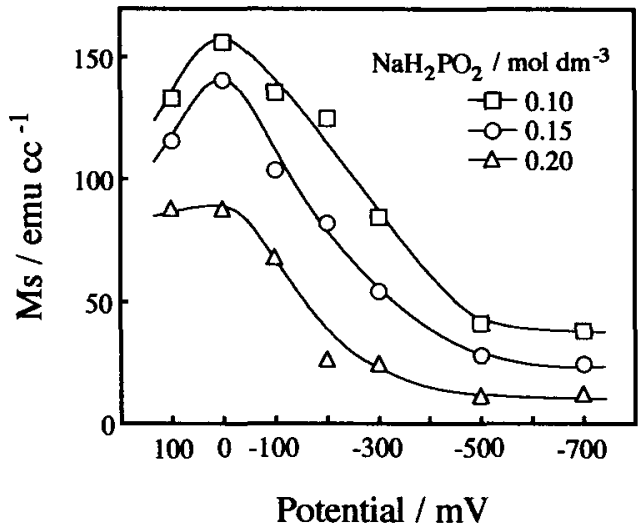

Fig. 5 Dependence of deposition potential on saturation magnetization of $\mathrm{NiP}$ films plated from various baths.

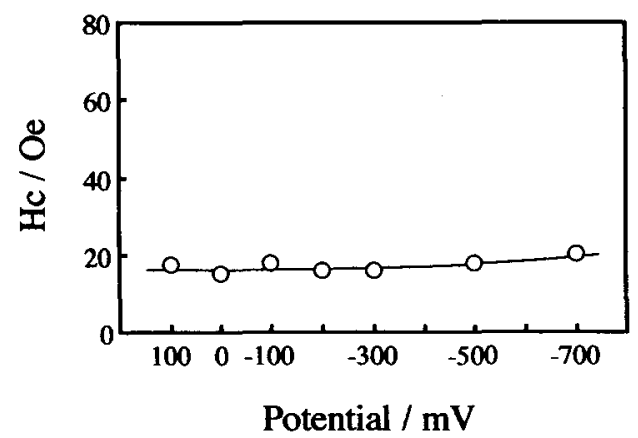

Fig. 6 Dependence of deposition potential on coercivity of NiP films.

from other plating baths showed almost identical results.

3.1.3 Microstructure and composition of the films

Further investigation was carried out in order to discuss the NiP films in detail. First, the crystallinity was examined. Only one diffraction peak from the (111) plane of fcc-nickel was observed in the $X$-ray diffraction patterns for all the Nip films fabricated by electroless plating with/ without electrolysis; therefore, it can be said that the crystallites in the films are oriented (111) plane parallel to the surface of the film. This result coincides with that of a previous reference ${ }^{7}$ ) reporting that the NiP alloy with a phosphorous content of from ca.2.5 up to $10 \mathrm{wt \%}$ shows a (111) orientation of fcc-nickel. The change in the (111) peak intensity upon electrolysis is plotted in Fig. 7a). There was no evident change in the peak intensity; moreover, no change was detected in the peak angle. Therefore, it is considered that no remarkable change occurred in crystallinity upon electrolysis. Although the films contain crystallites, the crystallinity is thought not to be very high, for the peak intensity was rather low and the peak was somewhat broad.

Next, the film compositions were measured. Figure 7b) shows the phosphorous content of the NiP films; the rest of the film is balanced with nickel. The phosphorous content was 3.6 wtx for the electrolessly plated NiP film; it increased to $8.1 \mathrm{wt} \%$ when cathodic polarization proceeded to $-700 \mathrm{mV}$. Nickel-phosphorous alloy containing this amount of phosphorous is regarded as a supersaturated solid solution of fccnickel with phosphorous ${ }^{8}$ ), which contains a


Fig. 7 Dependence of deposition potential on fcc Ni(111) peak intensity and P content of NiP films. 
large number of microscopic distortions 9 ). Another group has reported that crystallites get much smaller when nickel contains several percent of phosphorous ${ }^{10}$ ). These results also suggest that the crystallinity of the Nip films in this study was not very high because of the solution of phosphorous in the film. Phosphorous also decreases the magnetization of the film, acting as a nonmagnetic diluent. The effect is that phosphorous contributes as the solute to fill the $3 \mathrm{~d}$ band in the nickel11). The phosphorous in the NiP films in this paper seems to dilute the magnetization of the films in almost similar manner. The change in the saturation magnetization of the NiP films is thought to be due to the change in the phosphorous content of the films upon electrolysis. In other words, the phosphorous content of the NiP film can be controlled by applying electrolysis on electroless plating.

The tendency showed in Fig. 7b) -an increase in the phosphorous content with an increase in the cathodic polarizationwas contrary to that of electroplating 7,10$)$. Several hypotheses have been proposed regarding the state of phosphorous codeposited in nickel-phosphorous alloy $8,9,12,13)$. In any case, the reduction reaction of phosphorous which is accelerated by applying electrolysis should occur when phosphorous is deposited from a solution with anions which contain the phosphorous. The following mechanism may be assumed for a reduction (codeposition) reaction of phosphorous ${ }^{14-16)}$ in this paper: the acceleration of some reaction (such as a decomposition reaction of hypophosphite) of the electroless plating upon electrolysis, and the acceleration of a reduction reaction of phosphorous by electron supplied from simultaneous electrolysis. Although it is difficult to define the mechanism, one observer ${ }^{17)}$ has noted acceleration of the reaction of electroless plating upon the application of electrolysis, which implies the existence of the former mechanism.
Figure 8 shows the relationships between the phosphorous content and the saturation magnetization for NiP films plated from similar series of baths. When films with the same phosphorous content are compared, the saturation magnetization of the films plated with simultaneous electrolysis is found to be lower than that of the films plated electrolessly 6 ). Therefore, the NiP films formed by the electroless plating with electrolysis seem to have a somewhat homogeneous(less segregated) structure compared with those formed by electroless plating. The contribution of simultaneous electrolysis (which increases the phosphorous content) to electroless plating may become smaller for phosphorous-rich area, because the conductivity of the area may be lower. The difference in the segregation must be explicable by assuming the existence of such a mechanism.

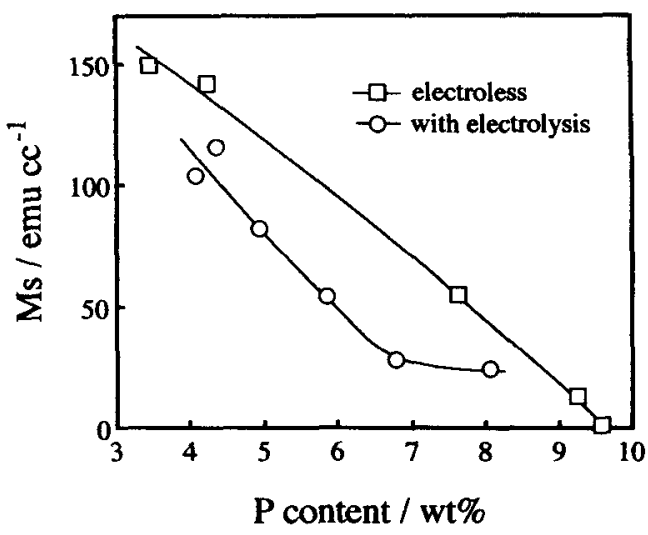

Fig. 8 Dependence of $\mathbf{P}$ content on saturation magnetization of NiP films plated with/without electrolysis.

As for the coercivity, the reversal mechanism of magnetization may be dominated by wall motion in such soft-magnetic films. Accordingly, the coercivity is seriously influenced by the degree of difficulty in wall motion caused by internal stress and any impurity or defect existing in the matrix 18). Phosphorous, non-magnetic 
impurity in the film, increased and lesssegregated with the cathodic electrolysis. We should also take into account the internal stress of the film with such state of the phosphorous in order to denote the origin of the constant coercivity shown in Fig. 6.

\subsection{Application of pulsating current}

The changes in the saturation magnetization of the NiP films plated from the baths with various nickel sulfate concentrations and with various sodium hypophosphite concentrations upon pulse electrolysis are



$\mathrm{NiSO}_{4}$ conc. $/ \mathrm{mol} \mathrm{dm}^{-3}$

Fig. 9 Dependence of $\mathrm{NiSO}_{4}$ concentration on saturation magnetization of NiP films plated from various processes.

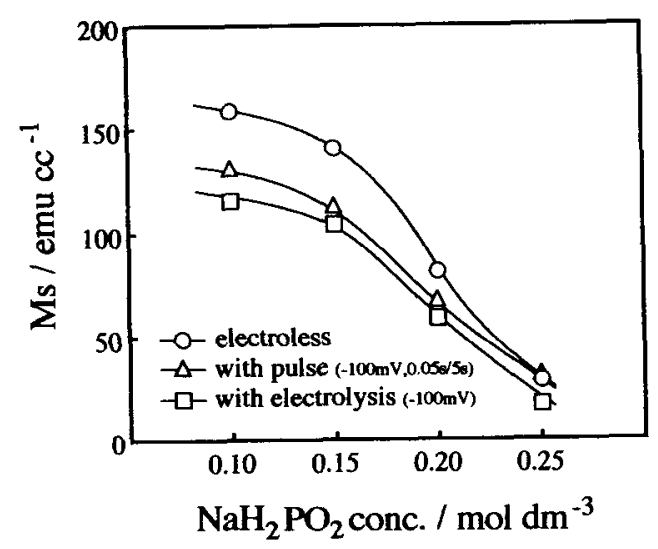

Fig. 10 Dependence of $\mathrm{NaH}_{2} \mathrm{PO}_{2}$ concentration on saturation magnetization of NiP films plated from various processes. plotted in Figs. 9 and 10 for various choices of conditions for simultaneous electrolysis as parameters. Because the applied voltage, $-100 \mathrm{mV}$, was not very high, no large change was observed in any case. Nevertheless, the change for the pulse electrolysis was close to that for the D.C. electrolysis, in spite of such a short pulse. It has been reported that nucleation begins with a pulse of several tens of $\mathrm{mV}$, less than geveral msec ${ }^{19}$ ), suggesting that the nucleation may occur with the cathodicpulse polarization. Such a change in the saturation magnetization observed upon the short-pulse electrolysis might be due to the remnant of the effect of electrolysis on the deposition mechanism of electroless plating, which also continues during the off-time of the pulse.

\section{CONCLUSION}

Based on the study outlined in this paper, the following conclusions may be drawn:

1. The NiP films with various saturation magnetizations could be fabricated by electroless plating with simultaneous electrolysis; this suggests that the application of electrolysis is very effective in controlling the properties of NiP films. The same tendency was observed in various NiP films plated from various baths.

2. The decrease in saturation magnetization upon electrolysis was due to the increase in the phosphorous content. This phenomenon, contrary to that in electroplating, was due to the acceleration of the codeposition reaction of phosphorous in electroless plating upon simultaneous electrolysis.

3. No large changes were observed in either the coercivity or the crystallinity of the Nip films.

4. It is suggested that the NiP films formed by the electroless plating with electrolysis have a somewhat homogeneous(less segregated) structure compared with those formed by electroless plating.

5. The magnetic properties also change with simultaneous pulse electrolysis. 
The authors are deeply indebted to Prof. Dr. Yukio Ichinose and Assoc. Prof. Dr. Takashi Ishiguro, Nagaoka Univ. Tech., for the measurement of the magnetic properties. It is also a pleasure to acknowledge the hospitality and encouragement of $\mathrm{Mr}$. Kazunori Hodouchi, N.U.T.

\section{REFERENCES}

1) T.Sakata and H.Honma, Hyomen Gijutsu, 40, 488 (1989).

2) H.Matsubara, K.Matsukawa, K.Tsuru and A.Yamada, Denki Kagaku, 60, 666 (1992).

3) A.Brenner and G.E.Riddell, J. Res. Nat. Bur. Std., 37, 31 (1946).

4) A.W.Goldenstein, W.Rostocker, F.Schossenberger and G.Gutzeit, J. Electrochem. Soc., 104, 104 (1957).

5) J.P.Marton and M.Schlesinger, J. Electrochem. Soc., 115, 16 (1968).

6) T.Osaka and I.Koiwa, Kinzoku Hyomen Gijutsu, 34, 330 (1983).

7) T.Watanabe and T.Kanayama, Hyomen Gijutsu, 40, 425 (1989).

8) A.H.Graham, R.W.Lindsay and H.J.Read, J. Electrochem. Soc., 112, 401 (1965).

9) K.Masui, S.Maruno and T.Yamada, Nihon Kinzoku Gakkaishi, 41, 1130 (1977).

10) K.Masui, T.Yamada and Y.Hisamatsu, Kinzoku Hyomen Gijutsu, 31, 611 (1980).

11) P.A.Albert, Z.Kovac, H.R.Lilienthal, T.R.McGuire and Y.Nakamura, J. Appl. Phys., 38, 1258 (1967).

12) J.P.Randin and H.E.Hintermann, J. Electrochem. Soc., 115, 480 (1968).

13) K.Masui, T.Nomura, S.Kwon and D.Chang, Hyomen Gijutsu, 43, 185 (1992).

14) G.Gutzeit, Plating, 46, 1158, 1275, 1377 (1959), 47, 63 (1960).

15) W.Machu and S.Gendi, Metalloberflache, 13, (4), 97 (1959).

16) V.Meerakker, J. Appl. Electrochem., 11, 395 (1981).

17) H.Homma, T.Fujinami and H.Takagi, Hyomen Gijutsu, 41, 777 (1990).

18) L.Neel, Ann. Univ. Grenoble, 22, 299 (1946).
19) L.T.Lan, I.Ohno and S.Haruyama, Denki Kagaku, 51, 167 (1983). 